\title{
Fluid-induced seismicity: comparison of rate- and state- and critical pressure theory
}

\author{
Friedemann Wenzel ${ }^{*}$ (])
}

*Correspondence:

friedemann.wenzel@kit.edu

Karlsruhe Institute

of Technology (KIT),

Geophysical Institute,

Hertzstr. 16, 76187 Karlsruhe,

Germany

\begin{abstract}
Induced seismicity as generated by the injection of fluids in a homogeneous, permeable medium with faults with variable proximity to rupture conditions is simulated using the rate- and state-dependent frictional fault theory (RST) of Dieterich ( $\mathrm{J}$ Geophys Res 99(B2):2601-2618, 1994) and the critical pressure theory (CPT) developed by Shapiro (Fluid-induced seismicity, Cambridge University Press, Cambridge, 2015). In CPT, the induced local seismicity density is proportional to the pressure rate, limited by the Kaiser Effect, and apparently un-related to the tectonic background seismicity. There is no time delay between a change in pressure rate and seismicity density. As a more complex theory, RST includes a time delay between a pressure change and induced seismicity and it is proportional to the natural tectonic background seismicity. Comparing both modelling approaches at a fixed location, this delay can be significant, dependent on a'free' parameter that represents the lower threshold for pressure below which seismicity is not triggered. This parameter can be tuned so that the results of CPT and RST become similar. Approximations of the RST allow a new interpretation of the parameter 'tectonic potential' that controls the level of induced seismicity in CPT.
\end{abstract}

Keywords: Fluid-induced seismicity, Pressure diffusion, Rate- and state-dependent frictional fault theory, Critical pressure theory

\section{Background}

Gaucher et al. (2015) provide an overview on published methods employed for modelling induced seismicity in geothermal reservoirs. Here I focus exclusively on the rateand state-dependent theory of Dieterich (1994), further referred to as RST for modelling fluid injection induced seismicity. For simplicity, I consider the case of fluid injection at the origin in a medium with isotropic pressure diffusion, characterized by constant diffusivity. Pressure depends on time and distance to the injection point only. I compare the induced seismicity obtained by RST with results of the critical pressure theory (CPT) developed by Shapiro et al. (2005) and subsequent papers and summarized in Shapiro (2015). RST and CPT differ mainly in the following aspects: (1) CPT postulates a density of seismogenic faults to which induced seismicity is proportional. RST assumes a tectonic background seismicity to which induced seismicity is proportional. (2) A change of stress to an individual fault has different consequences in RST and CPT. CPT considers only changes in normal stress, which is modified by the pore pressure of the injected fluid. If this pressure exceeds the criticality that is attributed to the fault it

(c) The Author(s) 2017. This article is distributed under the terms of the Creative Commons Attribution 4.0 International License (http://creativecommons.org/licenses/by/4.0/), which permits unrestricted use, distribution, and reproduction in any medium, provided you give appropriate credit to the original author(s) and the source, provide a link to the Creative Commons license, and indicate if changes were made. 
will rupture immediately. RST includes normal and shear stress changes and models the fault response with the rate- and state-dependent friction theory which assumes the friction on the fault to change with time and eventually lead to instability (=rapid rupture) but not instantaneously with the change of stress. In rate- and state-dependent friction theory faults are never at rest, they always slip with time but change from geologic slow slip rates to catastrophic slip rates, which are called earthquakes. (3) For an ensemble of seismogenic faults, the rate- and state-depended friction behaviour demands a nonuniform distribution of initial slip rate conditions. Exposed to a constant tectonic shear stress rate, this initial condition generates the constant background seismicity. Exposed to a specific time-dependent stress history, a time-dependent seismicity evolves. Dieterich (1994) provides the theory and the respective formulae.

In RST, faults are always slipping with a slip rate (=speed of differential motion across the fault). The friction on the fault (relating normal and shear stress) depends on this slip rate and one or more state parameters. The temporal evolution of the state parameter depends on the slip history. However, there is a steady state, where friction does not change, if the slip rate is constant and normal stress does not change. In a springslider model, a shear stress change is the product of the spring stiffness and the slip, or conversely an applied shear stress history results in slip history on the fault. There is a critical slip size beyond which the fault 'runs away', e.g. the slip rate become very high and the system unstable. The notion of instability in RST is not a transition from no slip to slip but the transition from slow to fast slip. Roy and Marone (1996) show that in response to a step-wise increase of shear stress, a fault experiences first a phase of quasi-static motion when the inertial force is negligible and later an inertia-dominated phase where friction can be neglected, the slip rate becomes very high. In terms of fault friction, the sudden increase in shear stress causes an immediate increase in friction, followed by the quasi-static decrease of friction with slip rates above geologic slip rates but below the inertia-driven fast rate. Only the latter one constitutes the earthquake. Rateand state-dependent friction laws conform to laboratory observations (Linker and Dieterich 1992) and are required to model aftershocks, which was the initial application of RST. If shear stress is changed instantaneously by a main shock, the seismicity does not appear immediately in the area around the main fault where the stress change is large enough but evolves with time resulting in an Omori-type temporal distribution of seismicity (Omori 1894; Utsu 1961). This behaviour cannot be understood with a criticality model, as in this case all aftershocks would occur immediately after the main shock and not days, months, or years later.

Whereas RST requires a specific slip rate distribution to model a constant tectonic background seismicity that changes in response to a stress field superimposed on the tectonic shear loading, CPT requires assumptions on the spatial distribution of criticality only and includes no tectonic background seismicity. Whereas CPT is designed for changes in normal stress by changing pore pressure, Dieterich (1994) derives a general equation for seismicity changes, as compared to the background seismicity if shear and/ or normal stresses are changed. The RST has been used in the simulation of aftershocks and earthquake swarms (Catalli et al. 2008; Daniel et al. 2011; Dieterich et al. 2000; Kilb et al. 2002; Toda et al. 2002, 2003) and recently also utilized for seismicity modelling in geothermal reservoirs (Hakimhashemi et al. 2014). In this model, the change 
in Coulomb failure stress (King 2007) is combined with the RST theory. Segall and Lu (2015) reformulated the RST seismicity evolution equation and included the Coulomb failure stress to model the seismicity as consequence of dyke intrusion in volcanic systems. Dieterich et al. (2015) addressed the seismicity caused by fluid injection, however, without employing their seismicity formula. The CPT has emerged from the study of induced seismicity in relation to permeability for instance in (Shapiro et al. 1999, 2002; Rothert and Shapiro 2003; Shapiro and Dinske 2009).

The next section represents a summary of the essentials of the critical pressure theory (CPT) including the Kaiser Effect and the rate- and state-dependent theory (RST), which was originally set up for sudden change of shear stress (earthquakes) and has been modified for the case of pressure changes by Wenzel (2015). The evolution of seismicity can be written as a Ricatti Equation (Ince 1956, pp. 23-35), which is used for approximations as well as for numerical implementation. The approximations allow (1) to understand the similarity of results in modelling seismicity with CPT and RST, and (2) to establish a scaling relation between RST and CPT that leads to a new interpretation of the 'tectonic potential' that controls the level of induced seismicity in CPT.

It is important to understand the differences between the stress changes in modelling aftershocks and pressure-induced seismicity. Pressure affects normal stress on faults. Released shear stress, after an earthquake, mostly affects shear stress on faults in its vicinity. The main difference in terms of induced seismicity is that the latter is a sudden change in stress whereas the former is a smooth change provided by pressure diffusion. The implications of this, on RST seismicity is demonstrated in the section on 'Approximate behaviour of rate and state-dependent Ricatti solutions'. Induced seismicity after pressure shut-in is relevant as seismicity does not stop with shut-in; moreover, in many cases, the maximum magnitudes are observed. The post-shut-in seismicity is controlled by the diffusion constant of the pressure and the lower cut-off pressure below which no seismicity can be triggered. This applies to both theories and does not constitute a major difference. The implications for the seismicity evolution are studied numerically.

\section{Comparison of critical pressure theory and rate- and state-dependent theory}

I briefly review the key features of the critical pressure theory (CPT) for induced seismicity. A permeable medium contains faults with density (faults per unit volume) $n_{f}(\vec{x})$ with location vector $\vec{x}$. The fault density is understood as the density of seismogenic faults. There may be faults that slip in an aseismic way but they are not subject of induced seismicity. Faults have different sizes and it is often assumed that the size distribution follows an exponential law so that the magnitude distribution in each source volume is compatible with a Gutenberg/Richter (1956) distribution. As seismicity is defined as the number of earthquakes within a source volume above a certain magnitude threshold, we set this magnitude threshold for simplicity of the mathematics to zero. If the actual threshold for lower magnitude, usually constrained by the magnitude level where seismic monitoring provides complete observations, is different, the fault density can easily be scaled from magnitude zero with the Gutenberg/Richter distribution. The injection of fluid into the medium is modelled as a diffusion process. The modelling of fault ruptures includes two aspects: (1) An individual fault can be triggered by pressure changes associated with fluid injection. The shear stress on these faults provided by the 
tectonic environment is characterized by a criticality parameter $C>0$; this value must be exceeded by the pore pressure to trigger an earthquake. According to Shapiro (2015, p. 202) the simplest assumption on the spatial distribution of seismogenic faults and criticality is that the faults are randomly distributed in space, statistically homogeneous so that the fault density fully characterizes their statistics. Each of these faults is associated with a criticality value. If it is high, the fault is rather stable and requires a high pore pressure during fluid injection to trigger it. If criticality is low, the fault is close to rupture condition and requires only a small pressure for triggering. The size of criticality is modelled by a distribution function and assumed to be independent on location. The cumulative distribution $F_{C}(C)$ is the probability that the criticality parameter is less than $C$. It is further assumed that there is a lower trigger threshold $C_{\mathrm{CPT}}$ (typically $1-100 \mathrm{kPa}$ ) below which no triggering occurs. It is also reasonable to assume an upper threshold $C_{\mathrm{CPT}}+\Delta C$ beyond which the fault ruptured even if it not pre-stressed. $\Delta C$ should be on the order of the stress drop typically released by earthquakes: 3-10 MPa.

If fluids are injected in a porous permeable medium beyond the prevailing hydrostatic pressure starting at time zero, the pressure spreads in the pores and develops a spatio-temporal distribution $p(\vec{x}, t)$. The diffusion results in a pattern where the pressure is highest at the injection point and decreases away from it. (2) The second aspect of fault triggering is the Kaiser Effect. Kaiser (1953) discovered experimentally that metals, when repeatedly loaded, have a memory of the previously experienced maximum stress level. In acoustic emission (AE) of repeatedly stressed rock samples the effect can be defined as the absence of $\mathrm{AE}$ until the previous stress level is exceeded. A first attempt to investigate the Kaiser Effect in a rock (sandstone) was made by Goodman (1963). In the later work by Kurita et al. (1979), the influence of water saturation and time delay between loading cycles on the Kaiser Effect were studied. In the context of the CPT, this means that faults cannot be recharged for rupture. All faults with criticality up to $p(\vec{x}, t)$ have been ruptured. All remaining faults have criticalities above this value and require higher pressures for initiation of rupture. Therefore, the process of seismicity generation in CPT is rate- and state-dependent as it depends on $\frac{\partial p(\vec{x}, t)}{\partial t}$ as well as on $p(\vec{x}, t)$. The assumption that faults are not recharged during injection periods seems to be plausible if I assume that recharging occurs at tectonic strain rates with very long durations-typically hundreds of years. The rate dependency is reflected by the fact that seismicity density is proportional to the pressure rate and drops to zero once the rate becomes zero. The state dependency manifests itself in the upper and lower criticality bounds and in the Kaiser Effect. The seismicity emerges from the space-dependent fault density $n_{f}(\vec{x})$ by integration over space $\left(d^{3} \vec{x} \rightarrow \mathrm{d} V\right)$. It represents the number of events per unit time with magnitudes above zero for the entire volume that is affected by the pressure diffusion. With a lower threshold for triggering seismicity, the volume is finite but depends on time

$$
\dot{N}(t)=\int n_{f}(\vec{x}) \cdot f_{C}(p(\vec{x}, t)) \cdot \frac{\partial p(\vec{x}, t)}{\partial t} \mathrm{~d} V .
$$

Integration extends only on regions where $\frac{\partial p(\vec{x}, t)}{\partial t}$ is positive and, in addition, the Kaiser Effect must be considered. If the probability density function is assumed to be flat 
$\left(f_{C}=1 / \Delta C\right)$ and for a step-wise injection at a point the solution for the seismicity is the constant value

$$
\dot{N}(t)=\frac{n_{f}}{\Delta C} \cdot \frac{\dot{Q}_{0}}{S} .
$$

For time-variable fluid injection rates, the seismicity has to be calculated by convolving the input pressure signal with the Green's Function for the diffusion in a homogeneous 3D medium and the spatial integration must be done numerically. However, (2) is very useful for scaling CPT and RST.

I use a two-cycle pressure injection at the origin into a medium with constant diffusion coefficient $D$ to demonstrate main features of CPT. Figure 1 shows the volume flow rate time history at the injection point (with $20 \mathrm{l} / \mathrm{s}$ as maximum) and the pressure at 200, 300 , and $400 \mathrm{~m}$ calculated with the analytic solution of the Green's Function convolved with the source volume flow time history. With growing distance from the injection point $(r)$, the pressure amplitude becomes smaller. In addition, the diffusion process with $D=0.1 \mathrm{~m}^{2} / \mathrm{s}$ modifies the cyclic behaviour significantly at distances in excess of $100 \mathrm{~m}$. This follows from the relation of characteristic distance and time in diffusion $r^{2}=D \cdot \tau$ with the period of the pressure cycle as characteristic time. The pressure peaks at $200 \mathrm{~m}$ are shifted by $30 \mathrm{~h}$, at $300 \mathrm{~m}$ by $45 \mathrm{~h}$. The second maximum is larger than the first one, and at $400 \mathrm{~m}$ there is no distinct first peak and the second is shifted by $70 \mathrm{~h}$.

The induced seismicity is generated in the entire volume affected by fluid diffusion and must be evaluated by (1). Figure 2 (left panel) shows the result of the integration if the Kaiser Effect is ignored and a lower trigger threshold of $C_{\mathrm{CPT}}=1 \mathrm{kPa}$ is applied. The seismicity displays two cycles that mimic the input flow signal quite closely, with the second maximum slightly reduced as compared to the first one because of $C_{\mathrm{CPT}} \neq 0$. The Kaiser Effect modifies this substantially as shown in the right panel of Fig. 2. Whereas

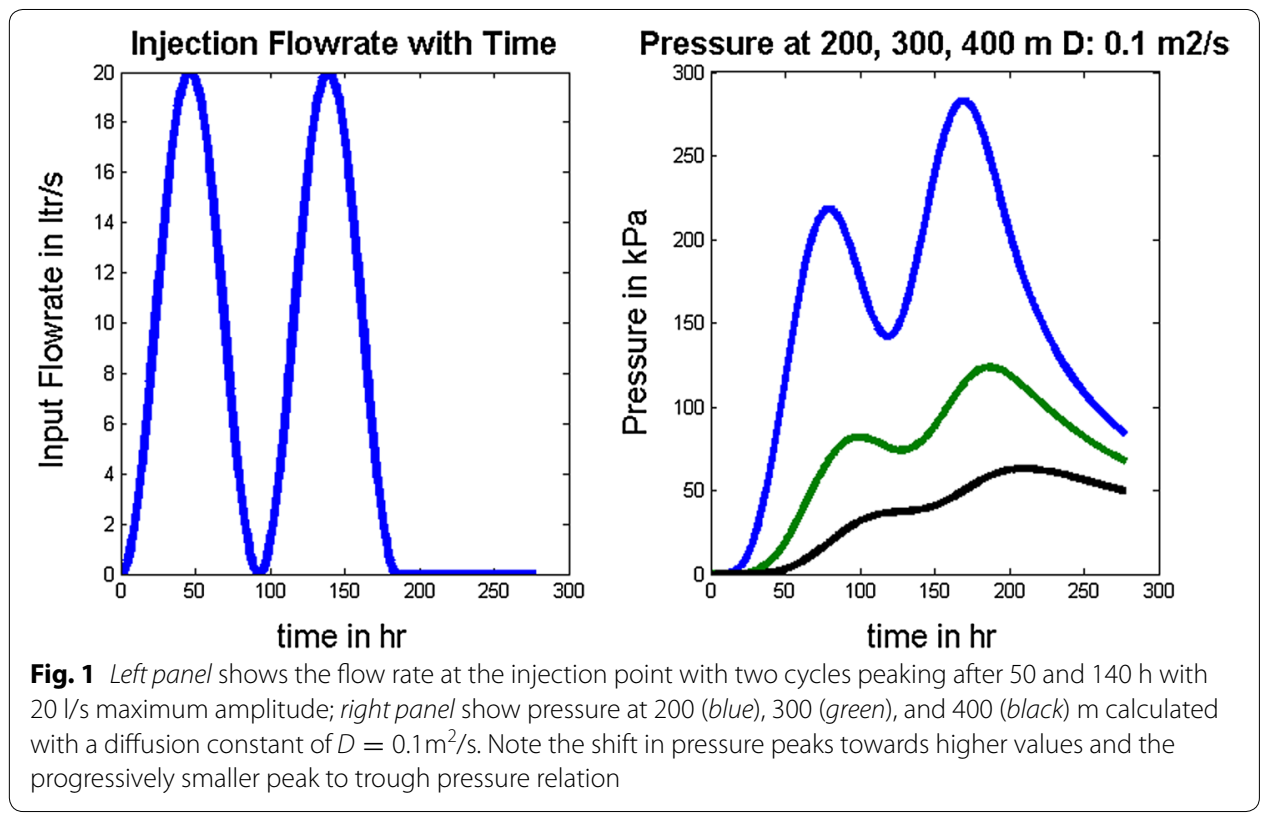



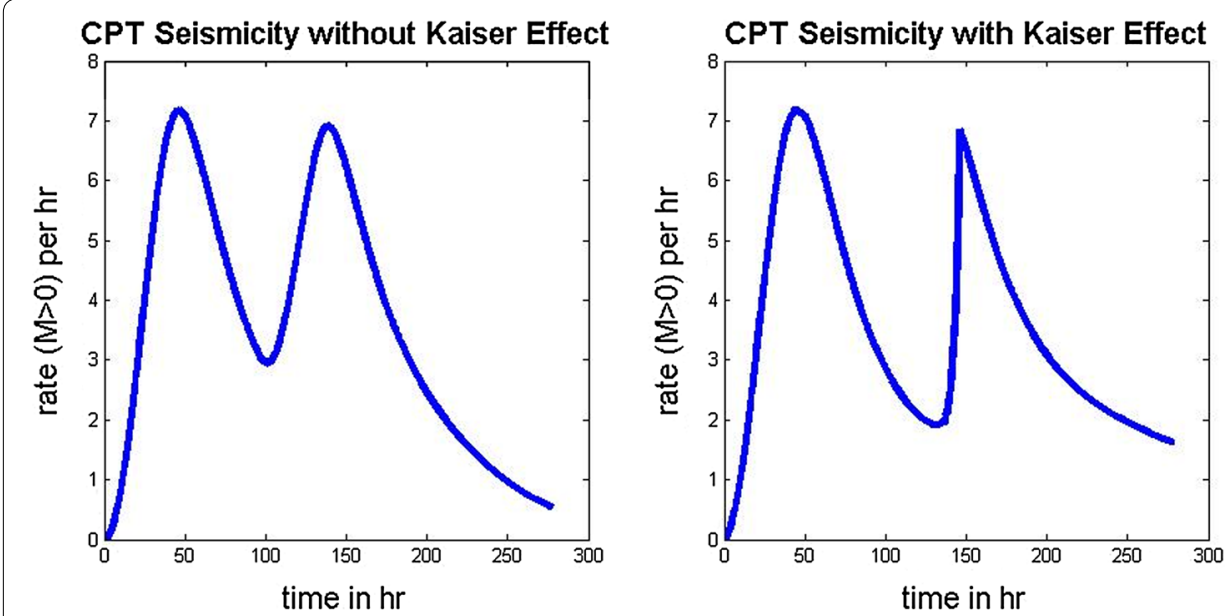

Fig. 2 Left Seismicity evolution with CPT ignoring the Kaiser effect. Note that it shows almost identical periodicity as the injection pressure. Right Seismicity evolution with CPT and considering the Kaiser effect. The early (until $80 \mathrm{~h}$ ) and late (after $150 \mathrm{~h}$ ) seismicity is not affected by the Kaiser effect, contrary to the time between 80 and $150 \mathrm{~h}$

the seismicity until $80 \mathrm{~h}$ is identical the second cycle changes shape to a sharp peak; after $150 \mathrm{~h}$, the seismicity is similar to the case without Kaiser Effect.

As shown in Wenzel (2015), the RST, originally developed in Dieterich (1994), can be used for pressure changes by fluid injection resulting in

$$
\begin{aligned}
\frac{\mathrm{d} \gamma}{\mathrm{d} t} & =\frac{1}{C_{\mathrm{RST}}} \cdot\left(1+\frac{p(t)}{\sigma_{n}^{0}}-\gamma(t) \cdot\left(\dot{\tau}_{\mathrm{tec}} \cdot\left(1+\frac{p(t)}{\sigma_{n}^{0}}\right)+g_{0} \cdot \dot{p}(t)\right)\right) \\
g_{0} & =\frac{\tau_{\text {tec }}}{\sigma_{n}^{0}}-\alpha
\end{aligned}
$$

with tectonic shear stress $\tau_{\text {tec }}$ shear stress rate $\dot{\tau}_{\text {tec }}$ normal stress $\sigma_{n}^{0}$, and $\alpha=0.2$. In the example calculation shown later, I assume the following values: $\sigma_{n}^{0}=50 \mathrm{MPa}, \frac{\tau_{\text {tec }}}{\sigma_{n}^{0}}=0.6$ so that $g_{0}=0.4, \dot{\tau}_{\text {tec }}=5 \mathrm{~Pa} /$ year

The seismicity density (=the number of seismic events with magnitude 0 or larger per unit time and unit volume) is

$$
\dot{n}(t)=\frac{1}{\gamma(t)} \cdot \frac{\dot{\nu}_{\text {tec }}}{\dot{\tau}_{\text {tec }}}
$$

with background seismicity density for seismic events with magnitudes 0 or larger $\dot{v}_{\text {tec }}$. The total seismicity, the equivalent to (1), is the integral over the volume affected by pressure increase:

$$
\dot{N}(t)=\int_{V} \dot{n}(t) d V
$$

$C_{\mathrm{RST}}$ controls the lower pressure $p(t)$ below which no seismicity will be triggered. Its role in the evolution of seismicity is similar to the lower pressure threshold $C_{\mathrm{CPT}}$ in CPT. This is not obvious in Eq. (3) but will become evident later in the paper. The assumptions 
in deriving (3) are: The tectonic shear stress rate is much smaller than the pressure rates within the medium and pressure $p(r, t)$ is much smaller than the normal stress $\sigma_{n}^{0}$.

The differential equation for seismicity density can be written in terms of the inverse of the function $\gamma(t)$ :

$$
R(t)=\frac{1}{\gamma(t) \cdot \dot{\tau}_{\text {tec }}}
$$

where $R(t)$ is dimensionless and represents the factor by which the natural tectonic background seismicity density is enhanced by the acting pressure. It is related to the induced seismicity density by

$$
\dot{n}(t)=R(t) \cdot \dot{v}_{\text {tec }}
$$

Combining (3) and (5) results in a Ricatti Equation for $R(t)$

$$
\dot{R}=-b \cdot R^{2}+a \cdot R
$$

with

$$
\begin{aligned}
& b(t)=\frac{\dot{\tau}_{\mathrm{tec}}}{C_{\mathrm{RST}}} \cdot\left(1+\frac{p(t)}{\sigma_{n}^{0}}\right) \\
& a(t)=b(t)+\frac{g_{0}}{C_{\mathrm{RST}}} \cdot \dot{p}(t)
\end{aligned}
$$

with the solution

$$
R(t)=\frac{1}{\exp \left[-\int_{0}^{t} a(\vartheta) d \vartheta\right]+\int_{0}^{t} b(\tau) \cdot \exp \left[-\int_{\tau}^{t} a(\vartheta) d \vartheta\right] d \tau}
$$

for $t=0 R(0)=1$ and after a constant pressure level is achieved at say $p_{\infty}$, where $\dot{p}=0$ the induced seismicity density change should vanish $\dot{R}(t \rightarrow \infty)=1$. Wenzel (2015) shows that expression (8) can be approximated by

$$
R(t)=\frac{\dot{n}(t)}{\dot{v}_{\text {tec }}} \approx g_{0} \cdot \frac{\dot{p}(t)}{\dot{\tau}_{\text {tec }}}
$$

For the case of constant injection rate starting at time zero, the approximate RST solution becomes

$$
\dot{N}(t)=\frac{\nu_{\text {tec }}}{\dot{\tau}_{\text {tec }}} \cdot g_{0} \cdot \frac{\dot{Q}_{0}}{S}
$$

Comparison to (2) allows scaling the RST solution for seismicity to the CPT solution of seismicity. They coincide for constant injection rate if

$$
g_{0} \cdot \frac{\dot{\nu}_{\text {tec }}}{\dot{\tau}_{\text {tec }}}=\frac{n_{f}}{\Delta C}
$$

If I ignore $g_{0}$, as it is in the order of 1 -or alternatively-if it is absorbed in $\Delta C$ the simplified equation 


$$
\frac{\dot{v}_{\text {tec }}}{\dot{\tau}_{\text {tec }}}=\frac{n_{f}}{\Delta C}
$$

can be understood with the concept of the seismic cycle. This concept has been established originally by Reid (1906) who hypothesized that the long-term tectonic loading results in a quasi-periodic release of seismic energy. For the seismicity of a crustal volume this means that during a cycle time $T_{\text {cyc }}$ all seismogenic faults are ruptured and critically loaded once. The product of background seismicity rate (=number of earthquakes per unit time and unit volume with magnitudes larger than 0 ) and cycle time is the number of all earthquakes that can occur in the volume, which in turn is the number of all seismogenic faults with potential of earthquakes larger than magnitude zero. The product of the tectonic stress rate and cycle time is the static stress drop on the seismogenic faults due to rupture.

$$
\frac{\dot{\nu}_{\text {tec }}}{\dot{\tau}_{\text {tec }}}=\frac{\dot{\nu}_{\text {tec }} \cdot T_{\text {cyc }}}{\dot{\tau}_{\text {tec }} \cdot T_{\text {cyc }}}=\frac{n_{f}}{\Delta C}
$$

It is thus evident that $\Delta C$ represents the static stress drop of earthquakes, typically in the range of 3-10 MPa for tectonic events (Kanamori and Brodsky 2004). Shapiro et al. (2007) call the ratio of fault density to stress drop the 'tectonic potential' as it characterizes the tectonics of the region within which fluid injection is done. As I show it is identical to the ratio of tectonic shear stress rate to tectonic background seismicity density. Thus the 'tectonic potential' can be better understood and also measured by geophysical ad geodetic means.

CPT utilizes the seismogenic index (SI) defined as $\Sigma=\log _{10}\left[\frac{n_{F}}{\Delta C \cdot S}\right]$, which in terms of the RST becomes $\Sigma=\log _{10}\left[\frac{g_{0} \cdot \dot{v}_{\text {tec }}}{\dot{\tau}_{\text {tec }} \cdot S}\right]$. Fault density must be provided in $\mathrm{m}^{-3}$, storativity in $\mathrm{Pa}^{-1}$, and $\Delta C$ in $\mathrm{Pa}$. Once a value for the storativity $\left(S=10^{-11} \mathrm{~Pa}^{-1}\right.$ in the calculations) is assumed, a given SI requires a specific value of the background seismicity. For instance, $\Sigma=-1$ results in $\dot{v}_{\text {tec }}=0.125 \mathrm{~km}^{-3}$ year $^{-1}$. Figure 3 shows the

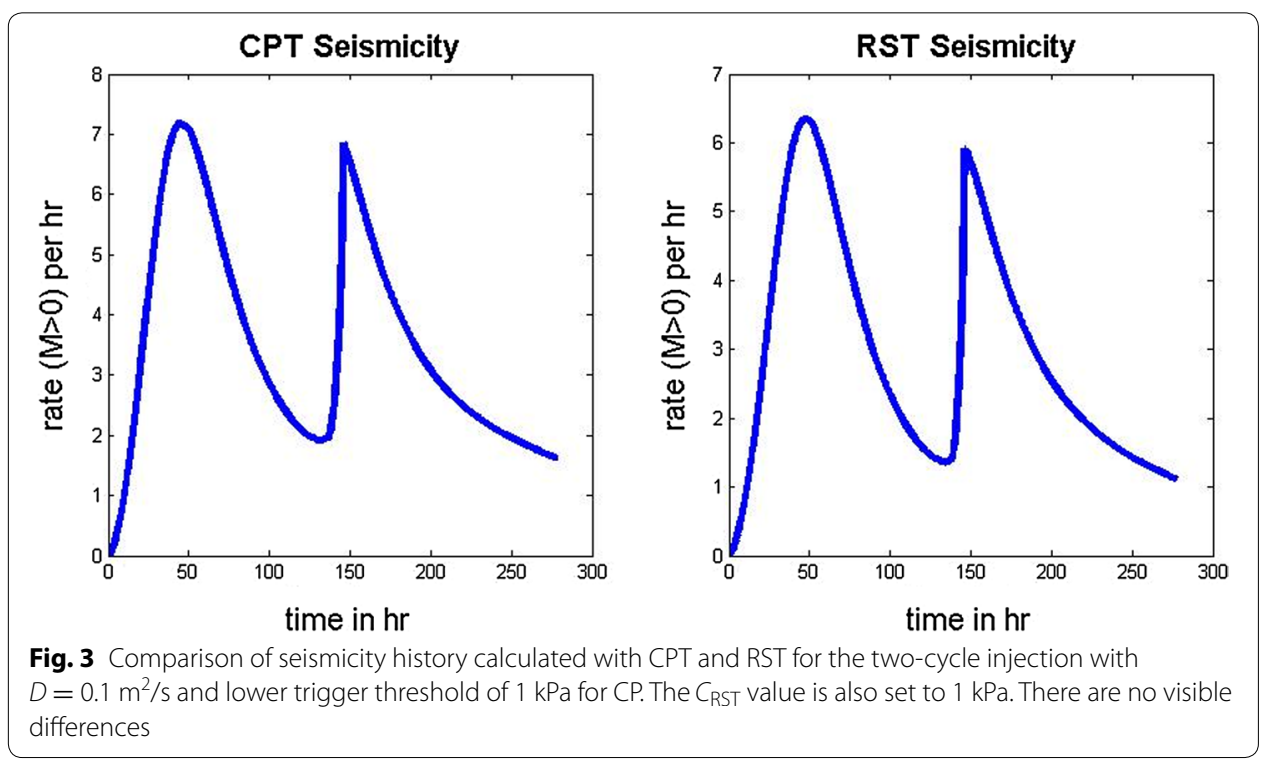


comparison between the CPT and RST results for total seismicity of the two-cycle injection with $D=0.1 \mathrm{~m}^{2} / \mathrm{s}$ (see Fig. 1). The lower trigger threshold for CPT is $1 \mathrm{kPa}$; and also $C_{\mathrm{RST}}=1 \mathrm{kPa}$. There are no differences visible. The comparison is surprisingly good given the circumstance that the methods as reflected in (2) and (4) are rather different.

\section{Approximate behaviour of rate- and state Ricatti solutions}

For specific pressure time histories, simple expressions for the resulting seismicity density can be derived. Here I consider (1) a step-wise sudden increase in pressure, (2) a constant pressure rate from zero to a higher level, and (3) the sudden drop of pressure rate from a high level. The first and second case is not realistic if the pressure diffuses smoothly into the medium. Even a step-wise increase of pressure at the injection point will become a smooth time history by the diffusion equation at close distances. However, as Dieterich (1994) studied a step-wise change of shear stress in the neighbourhood of a ruptured fault that generates aftershocks, it is interesting to see how induced seismicity emerges in response to a sudden change in pressure. If the pressure follows a Heaviside Function $p(t)=p_{0} \cdot H(t)$ the resulting approximate solution of the Ricatti equation is

$$
R(t) \approx \frac{\frac{g_{0} \cdot p_{0}}{C_{\mathrm{RST}}}}{1+\exp \left(\frac{g_{0} \cdot p_{0}}{C_{\mathrm{RST}}}\right) \cdot \frac{\dot{t}_{\mathrm{tec}}}{C_{\mathrm{RST}}} \cdot t}
$$

The approximation is valid if $p(t)<<\sigma_{n}^{0}=50 \mathrm{MPa}$ and $\frac{\dot{i}_{\text {tec }}}{C_{\mathrm{RST}}} \cdot t<<1$. The first condition is inherent in the derivation of (1) and the second condition is valid with $t$ limited to days. Expression (11) is similar to the seismicity density evolution derived in Dieterich (1994) for a step-increase of shear stress $\tau(t)=\tau_{0} \cdot H(t)$ if $g_{0} \cdot p_{0}$ is replaced by $\tau_{0}$. The solutions show an Omori-type of behaviour with Omori exponent being 1 . The seismicity density jumps to its highest level $\frac{g_{0} \cdot p_{0}}{C_{\mathrm{RST}}}$ immediately after time 0 . Then it decays to 0 with a time constant $t_{\mathrm{ST}}$ that can be defined as the time within which the initial seismicity level reduces to $50 \%$.

$$
t_{\mathrm{ST}}=\frac{p_{0}}{\dot{\tau}_{\mathrm{tec}}} \frac{\exp \left(-g_{0} \frac{p_{0}}{C_{\mathrm{RST}}}\right)}{\frac{p_{0}}{C_{\mathrm{RST}}}}
$$

For pressure steps with $\frac{p_{0}}{C_{\mathrm{RST}}}>>1$, the time is small because of the exponential function. In CPT seismicity density is proportional to the temporal derivative of the pressure. The response to a pressure following a Heaviside Function in time is a Delta Function, just a sudden burst in seismicity. The constant pressure rate represents the second case for local seismicity density evolution. Again, this is an idealized situation for pressure evolution controlled by the diffusion equation. However, it allows understanding the role of the parameter $C_{\mathrm{RST}}$ that appears in the solution of the Ricatti Equation. For the case of $p(t)=q \cdot t \quad 0 \leq t \leq t_{0}$ I find

$$
R(t)=\frac{q}{\frac{i_{\text {tec }}}{g_{0}}+q \cdot \exp \left(-\frac{g_{0}}{C_{\mathrm{RST}}} q t\right)}
$$

and for the seismicity density 


$$
\dot{n}_{\mathrm{RST}}(t)=\dot{v}_{\mathrm{tec}} \cdot R(t)=S \cdot 10^{\Sigma} \cdot \frac{q}{1+\frac{g_{0}}{\dot{t}_{\mathrm{tec}}} q \cdot \exp \left(-\frac{g_{0}}{C_{\mathrm{RST}}} q \cdot t\right)}
$$

The CPT solution of a pressure ramp until time $t_{0}$ with lower threshold $C_{\mathrm{CPT}}$ is

$$
\dot{n}_{\mathrm{CPT}}(t)=\left\{\begin{array}{cc}
0 & 0 \leq t \leq \frac{C_{\mathrm{CPT}}}{q} \\
S \cdot 10^{\Sigma} \cdot q & \frac{C_{\mathrm{CPT}}<t \leq t_{0}}{q} t_{0}<t \\
0 & t_{0}
\end{array}\right.
$$

CPT produces seismicity only if the temporal pressure rate is positive. The RST solution starts very small at $t=0$ and approaches the CPT solution with a time constant $t_{\mathrm{G}}$ that can be quantified as

$$
\dot{n}_{\mathrm{RST}}\left(t_{\mathrm{G}}\right)=\frac{1}{2} \dot{n}_{\mathrm{CPT}}(t) \text { so that } t_{\mathrm{G}}=\frac{C_{\mathrm{RST}}}{g_{0} \cdot q} \ln \left(\frac{g_{0} \cdot q}{\dot{\tau}_{\text {tec }}}\right)
$$

The delay time until seismicity becomes high is proportional to $C_{\mathrm{RST}}$. This justifies the understanding of this parameter as lower threshold of seismicity similar to $C_{\mathrm{CPT}}$. In addition, the delay time becomes progressively smaller with growing pressure rate $\mathrm{q}$. High pressure rate causes high seismicity density but small delay times. Conversely delay time manifests itself only when seismicity density is small. As small seismicity densities do not contribute significantly to the total seismicity history (see Eq. 2), the delay times inherent in RST are not very relevant and the good comparison between CPT and RST solutions as shown in Fig. 3 not surprising. The third case, the change in induced seismicity from a high level if the pressure drops and $\dot{p}(t)$ arrives at zero at time $t_{0}$ has been shown in Wenzel (2015) as being approximately

$$
R(t)=\frac{R\left(t_{0}\right)}{1+R\left(t_{0}\right) \frac{\dot{t}_{\mathrm{tec}}}{C_{\mathrm{RST}}} \cdot\left(t-t_{0}\right)} \quad t>t_{0}
$$

which represents again an Omori-type behaviour with decay time constant of seismicity $t_{D}=\frac{C_{\mathrm{RST}}}{R\left(t_{0}\right) \cdot \dot{\tau}_{\text {tec }}}$. The equivalent CPT solution is a sudden reduction to zero seismicity for times larger than $t_{0}$. The delay is again proportional to $C_{\mathrm{RST}}$. However, it becomes small if the seismicity density reflected by $R\left(t_{0}\right)$ is high at the time of pressure reduction. Similar to case (2), the delay time is more relevant for small seismicity density than for large one and consequently does not play a large role for the total seismicity history.

If $C_{\mathrm{RST}}$ is in the range of $\mathrm{kPa}$, the approximation of Eq. (9) is quite good and the Kaiser Effect is accommodated by the Ricatti Equation. The following example demonstrates the meaning of formulae (12) and (14) and visualizes the time constants $t_{\mathrm{G}}$ and $t_{D}$. I assume that at some location in the medium pore pressure increases linearly with $25 \mathrm{~Pa} / \mathrm{s}$ until a pressure of $10 \mathrm{MPa}$ is reached after $111 \mathrm{~h}$. After this the pressure remains constant. The $\mathrm{CPT}$ (with $C_{\mathrm{CPT}}=0$ ) predicts that there is an instantaneous seismicity density for $111 \mathrm{~h}$ and after this an immediate drop to zero. With the values for storativity and Seismogenic Index used before, the seismicity density with CPT is according to (13) $0.025 \mathrm{~km}^{-3} \mathrm{~s}^{-1}$. The lower threshold of CPT delays the onset of seismicity by $t=C_{\mathrm{CPT}} / q .1 \mathrm{kPa}$ as lower 


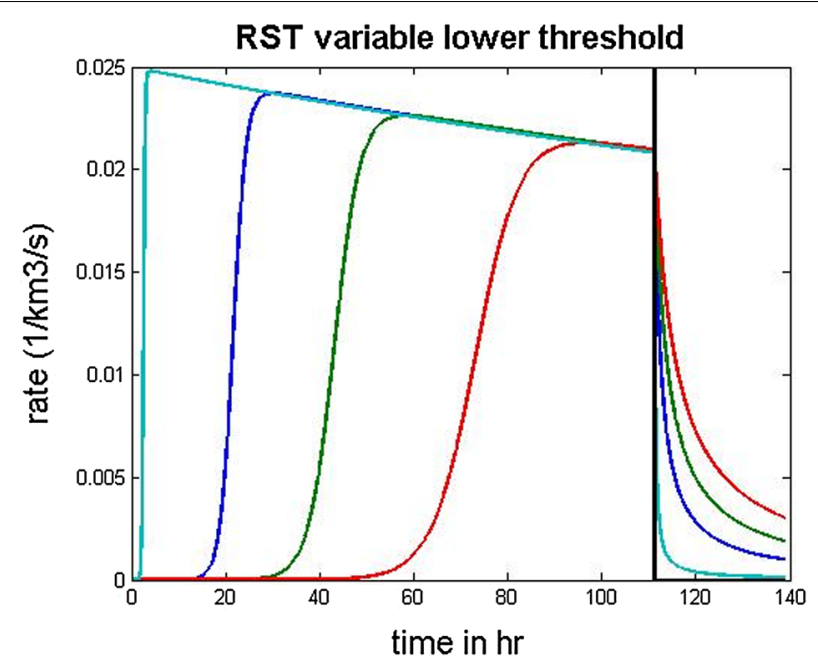

Fig. 4 Comparison between the CPT and RST seismicity density for a linear pressure ramp up to $10 \mathrm{MPa}$ at $111 \mathrm{~h}$ followed by a sudden drop of pressure rate to zero. CPT provides the constant seismicity of $0.025 /$ / $\mathrm{km}^{3} / \mathrm{s}$ until $111 \mathrm{~h}$ followed by a sudden drop to zero. RST depends very much on the lower threshold values $C_{\text {RST: }} 6,50,100,170 \mathrm{kPa}$ with time delays increasing with $C_{\text {RST }}$ during the time of pressure growth. After pressure rate drops to zero, RST seismicity falls off with a time constant again growing with $C_{\text {RST }}$

threshold causes only $40 \mathrm{~s}$ delay. Figure 4 shows the behaviour of RST for different values of the lower threshold. If I used the value of $1 \mathrm{kPa}$ for the threshold that has been used in Fig. 3, there would be no visible difference to the CPT response: an immediate increase as soon as the pressure starts to grow, and an immediate reduction to zero, once the pressure remains constant. For a value of $6 \mathrm{kPa}$, the seismicity density reaches the CPT level of $0.025 \mathrm{~km}^{-3} \mathrm{~s}^{-1}$ in a few hours, then shows a slight decay to $0.024 \mathrm{~km}^{-3} \mathrm{~s}^{-1}$ at $111 \mathrm{~h}$ and after the end of the pressure increase a rapid decay within a few hours. As expected from (12), the delay for seismicity to rise grows with growing lower threshold $(50,100,170 \mathrm{kPa})$ and the maximum seismicity densities become smaller. The time constants for the decay of seismicity, after $111 \mathrm{~h}$, are also proportional to the lower threshold. If I use the formulae for the time constants for growth and decay of seismicity, a threshold value of $C_{\mathrm{RST}}=100 \mathrm{kPa}$ provides $43 \mathrm{~h}$ until RST seismicity density grows to half the CPT level and $4 \mathrm{~h}$ to drop after $111 \mathrm{~h}$. These estimates match the full numerical solution of the Ricatti Equation in Fig. 4 well.

The key insight from the previous discussion is that if $C_{\mathrm{RST}}$ is in the range of $10 \mathrm{kPa}$ or even smaller the time constants involved in RST are quite small and do not influence the overall seismicity pattern strongly.

\section{Post-shut-in behaviour}

Post-shut-in pressure evolution is characterized by further diffusion of pressure deeper into the medium as compared to the pressure front at the moment of shut-in. Near the injection point, the pressure stops to grow so that its rate becomes negative. The consequence of further diffusion for the seismicity is that it does not stop with shutin although its rate slows down. As the volume affected by increasing pressure expands the potential size of rupture planes for earthquakes grows and thus the potential for higher magnitudes. This coincides with the observation that the highest magnitudes are 
frequently observed after shut-in (Shapiro and Dinske 2009; Shapiro et al. 2013). The pressure front of a constant point injection in a medium with homogeneous permeability can be characterized as the location where the pressure rate at a fixed distance has maximum size. As seismicity density is proportional to pressure rate it will be highest at these locations and thus serves as marker for the front, which is controlled by the diffusivity $D$ of the medium: $r_{F}(t)=\sqrt{6 \cdot D \cdot t}$ After shut-in, there is still a pressure front; however, its shape cannot be expressed analytically and in addition to $D$, it depends on the time of shut-in. The location where the pressure rate turns from positive values so that seismicity can be generated to negative values where no seismicity ceases is called the back front (Parotidis et al. 2004) and has the following form:

$$
r_{\mathrm{BF}}(t)=\sqrt{6 \cdot D \cdot t\left(\frac{t}{t_{\text {shut }}}-1\right) \ln \left(\frac{t}{t-t_{\text {shut }}}\right)} t>t_{\text {shut }}
$$

Seismicity at a specific time after shut-in can only occur within a spherical shell with the outer radius controlled by the seismicity front, and the inner radius by the back front. The actual size of the shell is controlled by the diffusivity and the lower threshold of the pressure $C_{\mathrm{CPT}}$ that must be exceeded before seismicity can be triggered. It causes a maximum distance beyond which not triggering will occur even if injection is maintained and thus has a significant influence on the outer radius but little influence on the back front (inner radius).

As formula (2) indicates, the level of seismicity before shut-in is independent on diffusivity, but would change with a lower threshold $C_{\mathrm{CPT}}$ required for the pressure before seismicity can be induced. The seismicity after shut-in, however, depends on both the injection rate and the diffusivity. In addition, $C_{\mathrm{CP}}$ has a significant influence. Langenbruch and Shapiro (2010) have studied the post-shut-in seismicity in the CPT context in detail and hypothesized an Omori-type decay of the seismicity after the end of fluid injection. A higher diffusivity causes a more rapid decay of seismicity. The diffusion front propagates faster for high diffusivity and thus reaches the lower threshold earlier in time. A higher threshold leads to an earlier stop and thus shortens the duration of seismicity after shut-in.

As an example, Fig. 5 shows the seismicity as calculated with CPT for the diffusivities 1.0 and $0.1 \mathrm{~m}^{2} / \mathrm{s}$ and for lower thresholds of $1 \mathrm{kPa}$ (blue), $5 \mathrm{kPa}$ (green), and $20 \mathrm{kPa}$ (red). Injection increases linearly during $50 \mathrm{~h}$ to $20 \mathrm{l} / \mathrm{s}$, remains constant for $20 \mathrm{~h}$, and after this drops to zero. The decay of seismicity after $70 \mathrm{~h}$ follows a power law as claimed by Langenbruch and Shapiro (2010). For given diffusivity, a higher threshold leads to a lower level of seismicity between $50 \mathrm{~h}$ and $70 \mathrm{~h}$ and a more rapid decay. This also applies to the lower diffusivity of $0.1 \mathrm{~m}^{2} / \mathrm{s}$. However, in this case, the role of the threshold becomes less significant. This is important for the assessment of post-shut-in seismicity. If a probabilistic estimate of the exceedance probability of a certain magnitude is demanded one needs to know the crustal volume that will be affected by pressure diffusion and the seismicity rate after shut-in. Both ingredients of hazard assessment depend on the lower threshold. The observation of the temporal and spatial evolution of seismicity during injection allows inferring the value of the diffusivity, but not the lower threshold value, which requires the observation of the decay of seismicity. Therefore, it appears difficult 


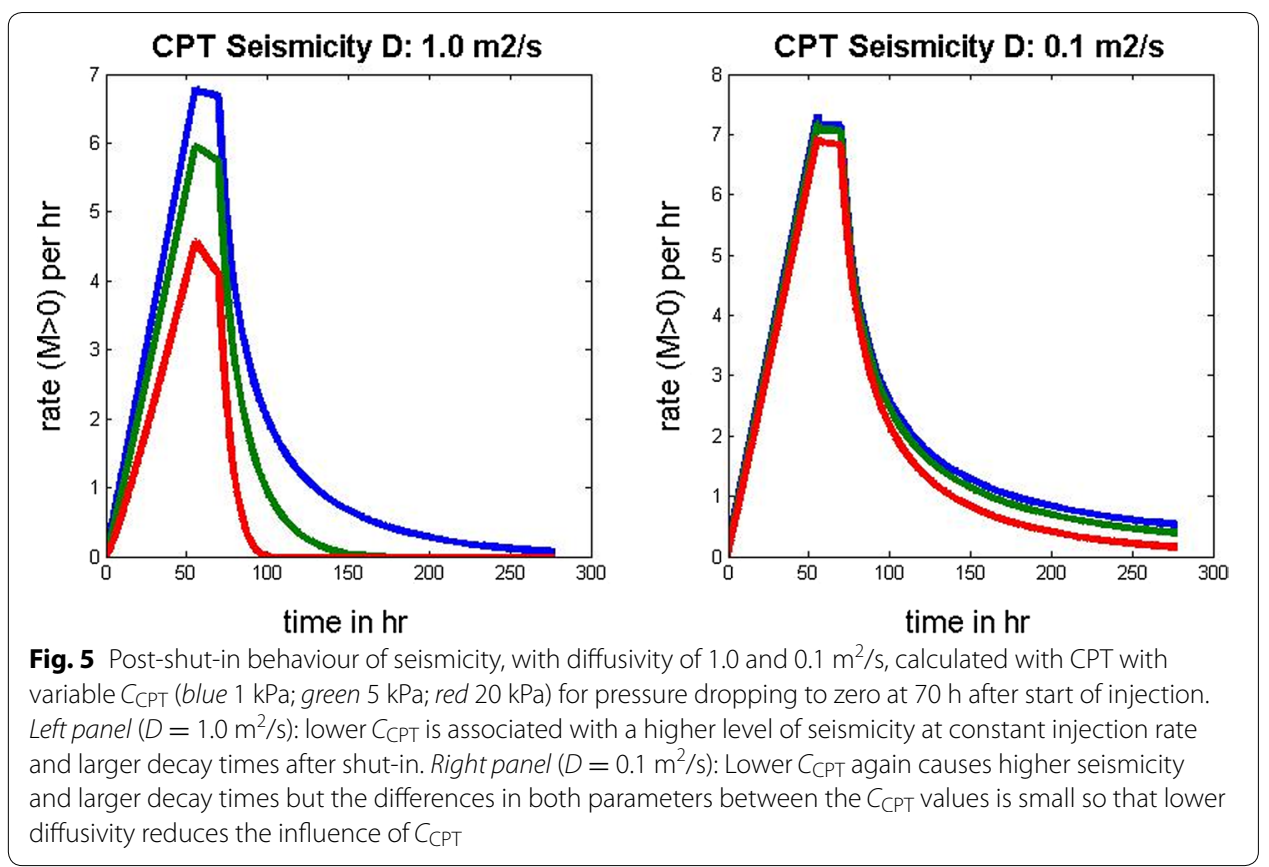

to assess the probability of post-shut-in earthquakes before the actual shut-in. However, for realistic diffusivities in rocks the dependency of post-shut-in earthquakes on the lower threshold becomes smaller and probabilistic assessments more feasible. As these aspects are not the topic of this paper, they are not discussed further.

The main topic, comparison of CPT and RST solutions in modelling post-shut-in behaviour, is addressed in Fig. 6. It compares the shut-in behaviour of CPT (left panel; identical to Fig. 5 left panel) and the RST solutions for $0.2 \mathrm{kPa}$ (blue), $1.0 \mathrm{kPa}$ (green),

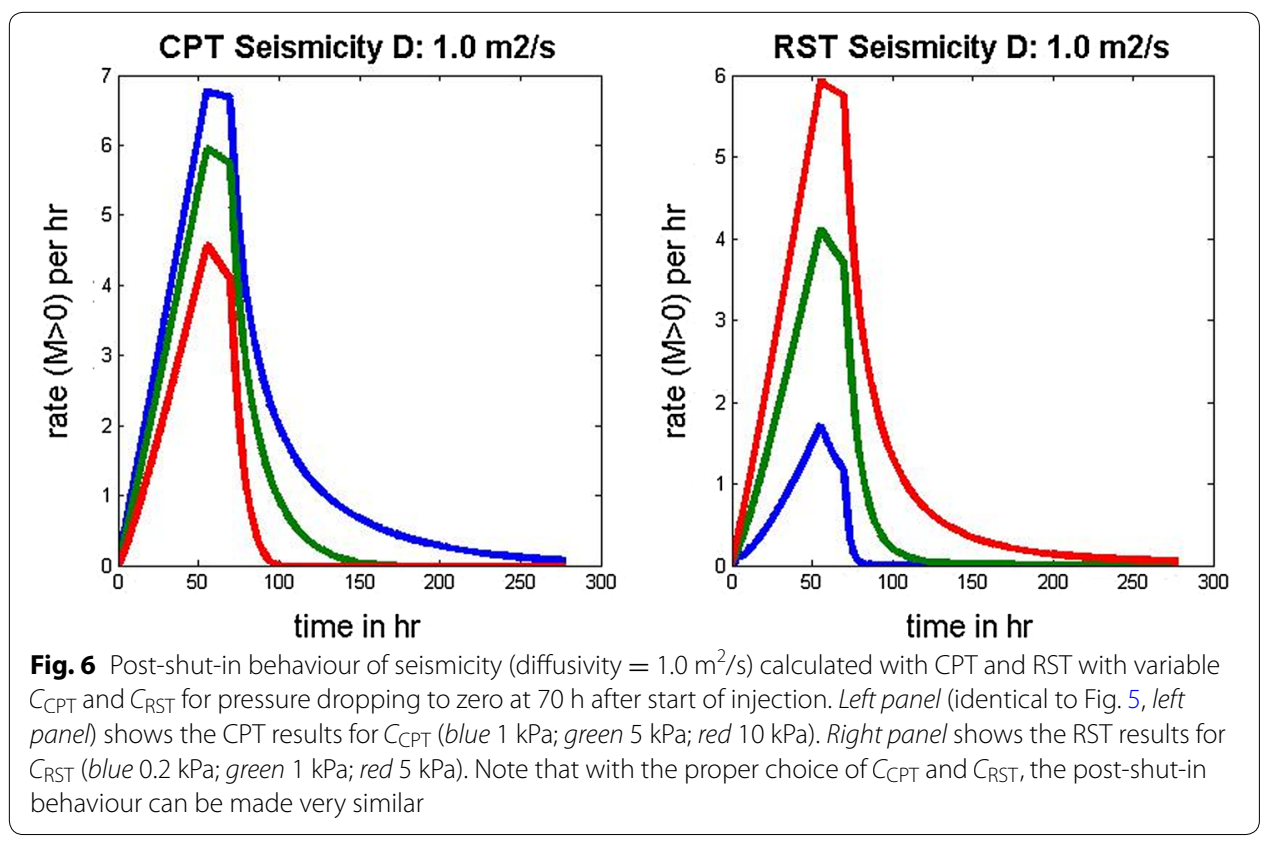


and $5.0 \mathrm{kPa}$ (red) values for $C_{\mathrm{RST}}$. The diffusivity is $1.0 \mathrm{~m}^{2} / \mathrm{s}$. For $0.2 \mathrm{kPa}$ (blue), the maximum seismicity is 6 events per hour and the tail after shut-in in the range of days. For $5 \mathrm{kPa}$ (red), the maximum value of seismicity is 1.8 events per hour but the tail is only in the range of hours. In general, a lower value of $C_{\mathrm{RST}}$ results in a higher level of maximum seismicity and a longer tail after shut-in. Conversely, a higher value results in a lower level of maximum seismicity and a longer tail after shut-in. In this sense RST, with $C_{\text {RST }}$ properly set, can produce very similar results as compared to CPT. Similar to what has been said earlier, it is possible to tune the lower threshold parameter for RST such that seismicity very similar to CPT evolves during the injection phase and after shut-in.

\section{Discussion and conclusion}

The critical pressure theory (CPT) developed by Shapiro (2015) for a quantitative description of the evolution of seismicity during fluid injection under pressure in permeable rocks assumes a Mohr-Coulomb-type rupture model for earthquakes. If the excess pressure exceeds the criticality of the fault, rupture occurs. This leads to a model where the seismicity density at a site is proportional to the temporal pressure derivative at this site. In addition, the Kaiser Effect must be considered. A quite different approach is provided by the rate- and state-dependent frictional fault theory (RST) of Dieterich (1994), which has been developed originally for modelling aftershock activity of larger earthquakes in response to sudden shear stress release. As RST has been formulated for shear and normal stresses acting on a medium, it can be modified for the case of fluid injection (Wenzel 2015) and results in a Ricatti Equation for the enhancement of the natural tectonic background seismicity at a site by fluid injection. The non-linear equation includes the Kaiser Effect and contains the time histories of pressure and its temporal derivative.

The comparison of both methods leads to the following conclusions:

Mathematical approximations allow simplifying RST to CPT solutions so that seismicity density becomes proportional to the temporal pressure derivative. With this results CPT can also be interpreted as enhancement of the natural tectonic background seismicity so that both theories predict a higher level of induced seismicity in areas where the natural seismicity is higher and conversely a lower level of induced seismicity in areas where the natural seismicity is lower.

Both theories include a parameter that controls the lower pressure threshold below which no seismicity is induced. The numerical comparison-without approximationsshows that the respective threshold value of RST can always be specified in a way that both RST and CPT provide very similar seismicity time histories for given injection time functions. This fact is established for the periods of active injections as well as for the shut-in phase. One can thus conclude that the computationally more demanding RST is not necessary for modelling-induced seismicity. The rate- and state-dependent friction behaviour of faults that is highly relevant in modelling aftershocks can be ignored in induced seismicity. This, in turn, is related to the key difference in stress histories to which a seismogenic medium is exposed in case of fluid injection and aftershocks. In the latter case, the medium experiences a stress shock as the shear stress release from the main earthquake occurs within seconds. In contrast, pressure changes resulting from a diffusion process are always 'smooth'; rate and state dependency is not relevant and 
occurs on short time scales and at minor rates of seismicity. Thus, CPT is a fine theory for modelling seismicity in an enhanced geothermal, i.e. petrothermal, reservoir.

\section{Authors' information}

Friedemann Wenzel is Prof. of Geophysics at Karlsruhe Institute of Technology (KIT). His main research fields are seismology, earthquake hazard, and risk.

\section{Acknowledgements}

I am indebted to two anonymous reviewers whose comments and suggestions helped significantly in improving the manuscript.

\section{Competing interests}

There are no potential competing interests. The paper has not been submitted anywhere else.

Availability of data and materials

Does not apply as no data are used; the study compares with synthetic examples.

\section{Publisher's Note}

Springer Nature remains neutral with regard to jurisdictional claims in published maps and institutional affiliations.

Received: 2 January 2017 Accepted: 30 May 2017

Published online: 08 June 2017

\section{References}

Catalli F, Cocco M, Console R, Chiaraluce L. Modelling seismicity rate changes during the 1997 Umbria-Marche sequence (central Italy) through a rate- and state-dependent model. J Geophys Res. 2008;113:B11301.

Daniel G, Prono E, Renard F, Thouvenot F, Hainzl S, Marsan D, Helmstetter A, Traversa P, Got JL, Jenatton L, Guiguet R. Changes in effective stress during the 2003-2004 Ubaye seismic swarm, France. J Geophys Res Solid Earth. 2011;116:B01309.

Dieterich J, Richards-Dinger K, Kroll K. Modeling injection-induced seismicity with the physics-based earthquake simulator RSQSim. Seismol Res Lett. 2015. doi:10.1785/0220150057.

Dieterich J, Cayol V, Okubo P. The use of earthquake rate changes as a stress meter at Kilauea volcano. Nature. 2000;408(6811):457-60.

Dieterich J. A constitutive law for the rate of earthquake production and its application to earthquake clustering. J Geophys Res. 1994;99(B2):2601-18

Gaucher E, Schoenball M, Heidbach O, Zang A, Fokker PA, van Wees JD, Kohl T. Induced seismicity in geothermal reservoirs: a review of forecasting approaches. Renew Sustain Energy Rev. 2015;52:1473-90.

Goodman RE. Subaudible noise during compression of rocks. Bull Geol Soc Am. 1963;74:487-90.

Gutenberg B, Richter CF. Magnitude and energy of earthquakes. Ann Geofis. 1956;9:1-15.

Hakimhashemi AH, Schoenball M, Heidbach O, Zang A, Grünthal G. Forward modelling of seismicity rate changes in georeservoirs with a hybrid geomechanical_statistical prototype model. Geothermics. 2014:52:185-94.

Ince EL. Ordinary differential equations. New York: Dover; 1956.

Kaiser J. Erkenntnisse und Folgerungen aus der Messung von Geräuschen bei Zugbeanspruchung von metallischen Werkstoffen. Archiv Eisenhüttenwesen. 1953;24(1/2):43-5.

Kanamori H, Brodsky EE. The physics of earthquakes. Rep Prog Phys. 2004;67:1429-96.

Kilb D, Gomberg J, Bodin P. Aftershock triggering by complete Coulomb stress changes. J Geophys Res. 2002. doi:10.102 9/2001JB000202.

King GCP. Fault interaction, earthquake stress changes, and the evolution of seismicity. Treatise Geophys. 2007:4:225.

Kurita K, Fujii N. Stress memory of crystalline rocks in acoustic emission. Geophys Res Lett. 1979;6:9-12.

Langenbruch C, Shapiro S. Decay rate of fluid-induced seismicity after termination of reservoir stimulation. Geophysics. 2010;75:MA53-62.

Linker MF, Dieterich JH. Effects of variable normal stress on rock friction: observations and constitutive equations. J Geophys Res. 1992;97:4923-40.

Omori F. On the aftershocks of earthquakes. J Coll Sci Imperial Univ Tokyo. 1894;7:111-200.

Parotidis M, Shapiro SA, Rothert E. Back front of seismicity induced after termination of borehole fluid injection. Geophys Res Lett. 2004. doi:10.1029/2003GL018987.

Reid HF. The mechanics of the earthquake, in the California earthquake of April 18, 1906, report of the state earthquake investigation commission II. Washington DC: Carnegie Institute; 1906.

Rothert E, Shapiro SA. Microseismic monitoring of borehole fluid injections: data modeling and inversion for hydraulic properties of rocks. Geophysics. 2003;68(2):685-9.

Roy M, Marone CH. Earthquake nucleation on model faults with rate- and state-dependent friction: effect of inertia. $J$ Geophys Res. 1996;101(B6):13919-32.

Segall P, Lu S. Injection-induced seismicity: poroelastic and earthquake nucleation effects. J Geophys Res Solid Earth. 2015; 120:5082-510

Shapiro SA. Fluid-induced seismicity. Cambridge: Cambridge University Press; 2015. 
Shapiro SA, Audigane P, Royer J. Large-scale in situ permeability tensor of rocks from induced microseismicity. Geophys J Int. 1999;137:207-13.

Shapiro SA, Dinske C. Scaling of seismicity induced by nonlinear fluid-rock interaction. J Geophys Res. 2009;1 14:B09307. doi:10.1029/2008JB006145.

Shapiro SA, Dinske C, Kummerow J. Probability of a given — magnitude earthquake induced by a fluid injection. Geophys Res Lett. 2007;34:L22314. doi:10.1029/2007GL031615.

Shapiro SA, Rentsch S, Rothert E. Characterization of hydraulic properties of rocks using probability of fluid-induced microearthquakes. Geophysics. 2005. doi:10.1190/1.1897030.

Shapiro SA, Rothert E, Rath V, et al. Characterization of fluid transport properties of reservoirs using induced microseismicity. Geophysics. 2002;67(1):212-20.

Shapiro AS, Krüger OS, Dinske C. Probability of inducing given-magnitude earthquakes by perturbing finite volumes of rocks. J Geophys Res. 2013;118:1-19. doi:10.1002/jgrb.50264.

Toda S, Stein R. Toggling of seismicity by the 1997 Kagoshima earthquake couplet: a demonstration of time-dependent stress transfer. J Geophys Res. 2003;108:2567. doi:10.1029/2003JB002527.

Toda S, Stein RS, Sagiya T. Evidence from the AD 2000 Izu islands earthquake swarm that stressing rate governs seismicity. Nature. 2002;419(6902):58-61.

Utsu T. A statistical study of the occurrence of aftershocks. Geophys Mag. 1961;30:521-605.

Wenzel F. Induced seismicity using Dietrich's rate and state theory and comparison to the Critical Pressure Theory, European Geosciences Union General Assembly 2015. EGU Energy Procedia. 2015;76(2015):282-90.

\section{Submit your manuscript to a SpringerOpen ${ }^{\circ}$ journal and benefit from:}

- Convenient online submission

\section{- Rigorous peer review}

- Immediate publication on acceptance

- Open access: articles freely available online

- High visibility within the field

Retaining the copyright to your article

Submit your next manuscript at $\boldsymbol{\nabla}$ springeropen.com 